\title{
Association of temporomandibular disorder with occupational visual display terminal use (Review)
}

\author{
HIDEO SHIGEISHI
}

\author{
Department of Oral and Maxillofacial Surgery, Institute of Biomedical and Health Sciences, \\ Hiroshima University, Minami-ku, Hiroshima 734-8553, Japan
}

Received March 10, 2016; Accepted April 14, 2016

DOI: $10.3892 /$ br.2016.669

\begin{abstract}
Increased visual display terminal (VDT) use has raised the prevalence of VDT-related adverse conditions, such as dry eye disease, and musculoskeletal and psychopathological symptoms, in office workers, including temporomandibular disorder (TMD). Many factors contributing to TMD have been identified, such as parafunctional habit (bruxism and teeth clenching), trauma, mental disorders, lifestyle, poor health, and nutrition, as well as hormonal factors (i.e., estrogen). It is likely that various contributing factors overlap in TMD development in individuals who routinely use a VDT for work. However, the relationship between TMD and VDT use has not been fully elucidated. In this mini-review, findings of recent studies of TMD in relation to occupational VDT use in Japan are discussed, as well as characteristic features and prevention strategies.
\end{abstract}

\section{Contents}

1. Introduction

2. VDT use-related disease

3. Prevalence of TMD in general population

4. Prevalence and etiology of TMD in individuals using a VDT at work

5. Prevention of TMD in occupational VDT users

6. Conclusion

Correspondence to: Dr Hideo Shigeishi, Department of Oral and Maxillofacial Surgery, Institute of Biomedical and Health Sciences, Hiroshima University, 1-2-3 Kasumi, Minami-ku, Hiroshima 734-8553, Japan

E-mail: shige@hiroshima-u.ac.jp

Abbreviations: TMD, temporomandibular disorder; VDT, visual display terminal

Key words: temporomandibular disorder, visual display terminal, occupational health

\section{Introduction}

Visual display terminals (VDTs) are used for a wide variety of work tasks, such as programming, data input, monitoring, and image construction. Increased VDT use has raised the prevalence of VDT-related disease, such as dry eye disease, and musculoskeletal and psychopathological symptoms, in office workers (1-3), with temporomandibular disorder (TMD) also reported as a VDT-related disease occurring in women who work at home as well as in a workplace environment (4).

TMD is a result of problems with the jaw, temporomandibular joint (TMJ), and surrounding facial muscles (5), while common signs and symptoms include restricted mouth opening, TMJ sounds, and muscle/TMJ pain. Such pain often leads to restricted jaw movements, resulting in difficulties with eating and speaking. Formerly, TMD was thought to occur as a result of malocclusion (5). However, though more recently several different influences, including parafunction (bruxism and teeth clenching), and traumatic, anatomical, psychological, hormonal (i.e., estrogen), and hereditary factors have been reported to be involved in its development (6-11). Among the risk factors for TMD, psychological aspects are thought to be important for understanding its etiology. It is considered that psychological conditions such as depression, anxiety, and stress may lead to the TMJ and masticatory muscles via sympathetic nerve activation, resulting in an increased risk of TMD onset and progression (4). Thus, it is expected that individuals with TMD-related symptoms are likely to be found in occupational fields. However, the relationship between TMD and occupational VDT use has not been fully elucidated. This review introduces recent studies of TMD in occupational settings in Japan to highlight its characteristics in association with VDT use.

\section{VDT use-related disease}

Individuals who use a VDT in an office environment typically sit in the same position for a long period of time, have repetitive and persistent finger movements, gaze at characters and images displayed by a VDT monitor, and are exposed to low levels of electromagnetic radiation $(12,13)$. According to a national survey conducted by the Japan Ministry of Health, Labour and Welfare, the percentage of individuals using a VDT for work tasks was only $15 \%$ in 1988 , which significantly increased to approximately $80 \% 10$ years later $(14,15)$. The 
rapid spread of VDTs in such devices as laptop computers, mobile phones, and portable information terminals, as well as generalization and long-time use, have led to VDT use-related diseases becoming diversified in recent years.

Ocular discomfort, musculoskeletal discomfort, and psychological problems are commonly experienced by VDT users working in many different industries (13). To eliminate the causes of VDT-related disorders, human engineering measures, such as improvements in posture, chair and keyboard design, education, and training, as well as regular rest breaks are required (13). Ocular discomfort is the most common VDT use-related symptom and approximately $60 \%$ of occupational VDT users have been reported to have eye pain and strain (16). Dry eye has also been observed in these workers, suggesting a close correlation between long durations of VDT use and dry eye (1). Furthermore, musculoskeletal symptoms such as stiffness and pain in the shoulders and neck have been observed in $30 \%$ of occupational VDT users (16), suggesting that the head and neck are the most common sites of musculoskeletal discomfort. In addition, approximately $40 \%$ of office workers studied in Bangkok reported musculoskeletal symptoms in the head and neck (2), while other areas of musculoskeletal discomfort include the upper back, waist, finger, arms, ankles, and feet, according to the specific job characteristics $(2,16)$.

In contrast to investigations of musculoskeletal symptoms, relatively few have been performed to clarify the associations between VDT usage at work and psychological problems (i.e., depression, anxiety and irritation). The percentage of individuals who use a VDT at work and have a mental disorder was previously thought to be relatively low as compared to those with psychological disturbances (12). However, the percentage of workers with mental health problems (i.e., strong anxiety, distress and stress) in a recent study conducted in Japan was found to be approximately $60 \%$, which was attributed to rapid generalization of VDT use in the workplace (17). Ye et al identified that $>5 \mathrm{~h}$ of VDT use at work each day was significantly associated with deterioration of mental health status and concluded that prolonged usage of a VDT for work-related tasks is a risk factor for mental disorders (18). In addition, companies in the information and communication industry in Japan showed the highest percentage (28.5\%) of employees who were absent from work for more than 1 month due to a mental health problem (17). These results suggest that mental health disorders have gradually increased to become a vital occupational health issue for heavy VDT users, and individuals in Japan working in an office environment.

\section{Prevalence of TMD in general population}

Studies conducted in the 1980s showed prevalence rates of TMD symptoms ranging from 16 to $59 \%$ in the general population (19). Such a wide range of prevalence may be attributed to the different survey methods used and composition of the subject groups examined. In a National Health Interview Survey conducted in the United States, the prevalence of TMD symptoms self-reported by adults was approximately 5\% in 2009 (20). In Japan, the National Survey of Dental Disease conducted in 2011 showed that the percentage of individuals (age, $\geq 15$ years) with TMJ sounds was approximately $15 \%$ in men and $17 \%$ in women, while the percentage of those with
TMJ pain was 2 and 4\%, respectively (21). Several studies performed in various countries have found that women have more TMD problems than men (22-24). Although a significant difference for total percentage was not observed between the two genders in Japan, women aged 25-29 years exhibited the highest prevalence of TMJ sounds and pain as compared to the other age groups (21). These results suggest that age is also a risk factor for prevalence of TMD.

\section{Prevalence and etiology of TMD in individuals using a VDT at work}

The prevalence of individuals who use a VDT at work and have TMD-related symptoms was found to be approximately $16 \%$ (25). Although it has been speculated that individuals who work in an office environment have a higher percentage of TMD-related symptoms as compared with the general population, the accurate rate of those who use a VDT in Japan has not been fully elucidated.

A variety of factors contribute to TMD, including parafunction (bruxism and teeth clenching), trauma, mental disorders, poor health and nutrition, and hormonal factors $(6-11,26)$. It is likely that various risk factors for TMD overlap, making the etiology complicated. Non-functional tooth contact induces excess tension, fatigue, and spasms in masticatory muscles, as well as overloading of the TMJ, resulting in TMD symptoms (27). In addition, TMJ pain was found to be associated with such behaviors as bruxism during sleep and clenching in office workers, suggesting that habitual behaviors may increase the risk of TMD (28). As for the correlation between TMD and psychological factors, office workers with TMD were reported to have higher levels of depression, anxiety, or continuous feelings of fatigue as compared to those without TMD (25). Mental health problems may independently influence the development and progression of TMD (29-31). Furthermore, significant correlations between mental health problems and bruxism/tooth clenching have been reported $(32,33)$, suggesting that psychological stress may have harmful effects on masticatory muscles and the TMJ by initiating non-functional tooth contact. Therefore, it is speculated that abnormal tooth contact and psychological disorders can lead to TMD in office workers.

As for individuals who use a VDT in occupational situations, Nishiyama et al reported that apparent TMD symptoms (limited mouth opening, mouth opening pain, mouth-opening deviation and chewing-induced pain) were statistically associated with the length of time working with a PC, indicating that VDT usage time is a significant risk factor for TMD (34). Notably, unconscious and continuous tooth contact during long periods of VDT use may have adverse effects on masticatory muscles and TMJ during the early onset stage of disease, resulting in the initiation of TMD. Furthermore, psychological factors such as depression, anxiety, and irritation may interact in a synergistic manner, leading to TMD with parafunction and interference with the normal healing process, resulting in the progression of TMD stage. Physiological changes induced by the sympathetic nervous system may cause decreased thresholds of pain and hyper-activation of the neuromuscular system, resulting in TMD-related musculoskeletal symptoms in individuals who use a VDT at work (4). Sympathetic nerve activation may be derived from a long period of work with a VDT, as well as 
environmental facts such as inappropriate desk height, and chair and computer mouse design, suggesting that work practice management should be effectively employed by use of a human engineering method. However, the major risk factors for the development of TMD in office workers remain largely unknown. A future epidemiological study may clarify the significant correlations of physical and mental problems with TMD in individuals who use a VDT in occupational settings.

\section{Prevention of TMD in occupational VDT users}

Occupational Health guidelines for use of a VDT at work published in 2002 by the Japanese Ministry of Health, Labor and Welfare include recommendations for properly adjusted monitor brightness, reduced glare and reflection, appropriate viewing angle, regular breaks (i.e., 10 min of rest for each hour) and correct working posture (13). Such control of the working environment and work practice management are essential to prevent VDT-related disease. In addition, health control factors, such as medical examinations, health consultations, and physical exercises, should be of use to improve physical and mental conditions (13). Since TMD is a multifactorial disorder, it is vital to understand the physical and psychosocial backgrounds of workers, including present and past illness, personal relationships at work, and family environment, as well as predisposing factors associated with occupational VDT usage. Previously reported effective self-care methods to prevent TMD include massage and exercises to relax the masticatory muscles, which were shown to be helpful for relieving excessive tension (35). Local hyperthermic treatments are also known to be effective to relieve masticatory muscle fatigue by promotion of blood circulation (36). To reduce continuous non-functional tooth contact during work, dentists should participate in changing the behavior of VDT users by teaching how to properly rest the teeth. As for bruxism during sleep at night, a mouth guard may help to relax masticatory muscles and the TMJ. Of note, TMD often develops in accordance with complicated and refractory problems, such as chronic pain disorders and unidentified complaints. Thus, it is necessary for dentists, physicians, mental health providers, pain management specialists, and other occupational experts to play a vital role in the prevention of TMD in relation to occupational VDT use.

\section{Conclusion}

Use of a VDT at work can contribute to a number of disorders, and is also a potential risk factor for TMD caused by physical and psychological stress. Dentists should educate VDT users regarding risk factors associated with TMD as well as how to relax the jaws. A detailed analysis of workers who use a VDT from physical, psychological, and social perspectives may be necessary for prevention and treatment strategies for TMD caused by occupational VDT use.

\section{References}

1. UchinoM,YokoiN,Uchino Y,DogruM,KawashimaM,Komuro A, Sonomura Y, Kato H, Kinoshita S, Schaumberg DA, et al: Prevalence of dry eye disease and its risk factors in visual display terminal users: The Osaka study. Am J Ophthalmol 156: 759-766, 2013.
2. JanwantanakulP, Pensri P, Jiamjarasrangsri V and Sinsongsook T: Prevalence of self-reported musculoskeletal symptoms among office workers. Occup Med (Lond) 58: 436-438, 2008.

3. Tarumi K, Nagami $M$ and Kadowaki I: An inquiry into the factors affecting the complaints of subjective symptoms in VDT operators. Sangyo Igaku 32: 77-88, 1990 (In Japanese).

4. Horowitz L and Sarkin JM: Video display terminal operation: A potential risk in the etiology and maintenance of temporomandibular disorders. Cranio 10: 43-50, 1992.

5. Al-Ani Z and Gray R: TMD current concepts: 1 . An update. Dent Update 34: 278-280, 282-284, 287-288, 2007.

6. De Boever JA: Functional disturbances of temporomandibular joints. Oral Sci Rev 2: 100-117, 1973.

7. Miyake R, Ohkubo R, Takehara J and Morita M: Oral parafunctions and association with symptoms of temporomandibular disorders in Japanese university students. J Oral Rehabil 31: 518-523, 2004.

8. Häggman-Henrikson B, Rezvani M and List T: Prevalence of whiplash trauma in TMD patients: a systematic review. J Oral Rehabil 41: 59-68, 2014.

9. Yap AU, Tan KB, Chua EK and Tan HH: Depression and somatization in patients with temporomandibular disorders. J Prosthet Dent 88: 479-484, 2002.

10. Berger M, Szalewski L, Bakalczuk M, Bakalczuk G, Bakalczuk S and Szkutnik J: Association between estrogen levels and temporomandibular disorders: a systematic literature review. Przegl Menopauz 14: 260-270, 2015.

11. Yoda T, Sato T, Abe T, Sakamoto I, Tomaru Y, Omura K, Hatano N, Takato T and Ishii Y: Long-term results of surgical therapy for masticatory muscle tendon-aponeurosis hyperplasia accompanied by limited mouth opening. Int J Oral Maxillofac Surg 38: 1143-1147, 2009.

12. Dy FJF: Visual Display Units: Job Content and Stress in Office Work, New Technologies and the Improvement of Data-Entry Work. International Labour Office, Geneva, Switzerland, 1985.

13. Japanese Ministry of Labour. Guidelines for Industrial Health Controls of VDT Operations. Roudoushou Kihatsu, Tokyo, 2002 (In Japanese)

14. Japanese Ministry of Labour: Survey on Technological Innovation and Labour/ Policy Planning and Research Department, Minister's Secretariat, 1988 (In Japanese).

15. Japanese Ministry of Labour: Survey on Technological Innovation and Labour/Policy Planning and Research Department, Minister's Secretariat, 1998 (In Japanese).

16. Iwakiri K, Mori I, Sotoyama M, Horiguchi K, Ochiai T, Jonai H and Saito S: Survey on visual and musculoskeletal symptoms in VDT workers. Sangyo Eiseigaku Zasshi 46: 201-212, 2004 (In Japanese).

17. Ministry of Health, Labor, and Welfare of Japan. Survey on State of Employees'. Health, 2012 (In Japanese).

18. Ye Z, Abe Y, Kusano Y, Takamura N, Eida K, Takemoto T and Aoyagi K: The influence of visual display terminal use on the physical and mental conditions of administrative staff in Japan. J Physiol Anthropol 26: 69-73, 2007.

19. Carlsson GE and LeResche L: Epidemiology of temporomandibular disorders. In: Temporomandibular Disorders and Related Pain Conditions (Progress in Pain Research and Management Series). Vol 4. 1st edition. Sessle BJ, Bryant PS and Dionne RA (eds). IASP Press, Seattle, pp211-226, 1995.

20. Slade GD: Epidemiology of temporomandibular joint disorders and related painful conditions. Mol Pain 10 (Suppl 1): O16, 2014.

21. Ministry of Health, Labour and Welfare of Japan. The survey of dental disease in 2012 (In Japanese).

22. Wänman A: Longitudinal course of symptoms of craniomandibular disorders in men and women. A 10-year follow-up study of an epidemiologic sample. Acta Odontol Scand 54: 337-342, 1996.

23. Dao TT and LeResche L: Gender differences in pain. J Orofac Pain 14: 169-184, discussion 184-195, 2000.

24. Johansson A, Unell L, Carlsson GE, Söderfeldt B and Halling A: Gender difference in symptoms related to temporomandibular disorders in a population of 50-year-old subjects. J Orofac Pain 17: 29-35, 2003.

25. Sugisaki M, Takano N, Kino K, Hayashi K, Saito T, Nishiyama A and Suzuki S: Prevalence of temporomandibular disorders among working population of Tokyo. Journal of the Japanese Society for the Temporomandibular Joint 20: 127-133, 2008 (In Japanese).

26. Parker MW: A dynamic model of etiology in temporomandibular disorders. J Am Dent Assoc 120: 283-290, 1990.

27. Funato M, Ono Y, Baba K and Kudo Y: Evaluation of the non-functional tooth contact in patients with temporomandibular disorders by using newly developed electronic system. J Oral Rehabil 41: 170-176, 2014. 
28. Tsukagoshi K, Nishiyama A, Kino K, Sugisaki M and Haketa T: The factors which affects temporomandibular disorders-related pain. Japanese Journal of Orofacial Pain 4: 47-55, 2011 (In Japanese).

29. Geissler PR: An investigation of the stress factor in the mandibular dysfunction syndrome. J Dent 13: 283-287, 1985.

30. Samant A, Martin JO and Cinotti WR: Psychologic factors influencing temporomandibular joint dysfunction: review of the literature. Compend Contin Educ Dent 7: 543-546, 1986.

31. Marbach JJ and Lipton JA: Biopsychosocial factors of the temporomandibular pain dysfunction syndrome. Relevance to restorative dentistry. Dent Clin North Am 31: 473-486, 1987.

32. Glaros AG, Williams $\mathrm{K}$ and Lausten L: The role of parafunctions, emotions and stress in predicting facial pain. J Am Dent Assoc 136: 451-458, 2005.
33. Renner AC, da Silva AA, Rodriguez JD, Simões VM, Barbieri MA, Bettiol H, Thomaz EB and Saraiva MC: Are mental health problems and depression associated with bruxism in children? Community Dent Oral Epidemiol 40: 277-287, 2012.

34. Nishiyama A, Kino K, Sugisaki M and Tsukagoshi K: A survey of influence of work environment on temporomandibular disorders-related symptoms in Japan. Head Face Med 8: 24, 2012.

35. Pierson MJ: Changes in temporomandibular joint dysfunction symptoms following massage therapy: a case report. Int J Ther Massage Bodywork 4: 37-47, 2011.

36. Nelson SJ, dos Santos J Jr, Barghi N and Narendran S: Using moist heat to treat acute temporomandibular muscle pain dysfunction. Compendium 12: 808-816, 810, 812 passim, 1991. 